\title{
An Extensive Comparison of Quantitative Trait Loci Mapping Methods
}

\author{
A. Kleensang $^{\mathrm{a}} \quad$ D. Franke ${ }^{\mathrm{a}} \quad$ A. Alcaïs $^{c} \quad$ L. Abel ${ }^{\mathrm{c}} \quad$ B. Müller-Myhsok ${ }^{\mathrm{b}} \quad$ A. Ziegler $^{\mathrm{a}}$ \\ a Institut für Medizinische Biometrie und Statistik, Universität zu Lübeck, Universitätsklinikum Schleswig-Holstein, \\ Campus Lübeck, Lübeck, and b Max-Planck-Institut für Psychiatrie, Computational Genetics Group, München, \\ Germany; ${ }^{C}$ Human Genetics of Infectious Diseases, INSERM/Université Paris 5 - Unité 550, Faculté de Médecine \\ Necker, Paris, France
}

\section{Key Words}

QTL $\cdot$ Monte-Carlo simulation study $\cdot$ Linkage $\cdot$ Type I error · Empirical power

\begin{abstract}
Background: The choices of study design and statistical approach for mapping a quantitative trait (QT) are of great importance. Larger sibships and a study design based upon phenotypically extreme siblings can be expected to have a greater statistical power. On the other hand, selected samples and/or deviation from normality can influence the robustness and power. Unfortunately, the effects of violation of multivariate normality assumptions and/or selected samples are only known for a limited number of methods. Some recommendations are available in the literature, but an extensive comparison of robustness and power under several different conditions is lacking. Methods: We compared eight freely available and commonly applied QT mapping methods in a Monte-Carlo simulation study under 36 different models and study designs (three genetic models, three selection schemes, two family structures and the possible effect of deviation from normality). Results: Empirical type I error fractions and empirical power are presented and explained as a whole and for each method separately, followed by a thorough discussion. Conclusions: The results from this extensive comparison could serve as a valuable source for the choice of the study design and the statistical approach for mapping a QT.

Copyright $\odot 2010$ S. Karger AG, Basel
\end{abstract}

\section{Introduction}

Over the last two decades complex traits with quantitative intermediate traits gained considerable interest in genetic epidemiology since many common diseases can be measured directly on a quantitative scale. These quantitative intermediate traits have been shown to be more powerful than a dichotomous disease definition [1], and many different approaches that are specifically designed for detecting linkage between genetic markers and a quantitative trait (QT) locus have been proposed quite recently. As stated by Blangero [2], the harvest has come: hundreds of chromosomal regions influencing traits like body weight [3], body stature [4], bone mass density [5, 6] or mild malaria [7] have been identified, and several human genes influencing QTs have already been mapped (see, e.g., [8]).

The choice of the study design and the choice of the statistical approach for mapping a QT are of great importance [9]. Most QT studies for linkage are based on nuclear families with either independent sib-pairs, i.e., one sib-pair per family, or possibly large sibships, i.e., more than one sib-pair per family. Since it is well established that larger sibships are more powerful then small ones for quantitative trait loci (QTL) mapping, though unfortunately only some methods are originally developed or ex-

\section{A. Kleensang and D. Franke contributed equally to the work.}

\section{KARGER}

Fax +4161306 1234 E-Mail karger@karger.ch www.karger.com

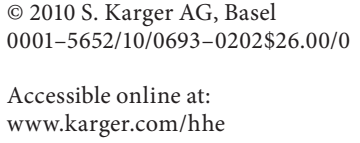

Prof. Dr. rer. nat. Andreas Ziegler

Institut für Medizinische Biometrie und Statistik

Universität zu Lübeck

DE-23538 Lübeck (Germany)

Tel +49 451500 2780, Fax +49 451500 2999, E-Mail ziegler@ imbs.uni-luebeck.de 
Table 1. Overview of used quantitative-trait linkage analyses methods, used abbreviations and programs with version numbers

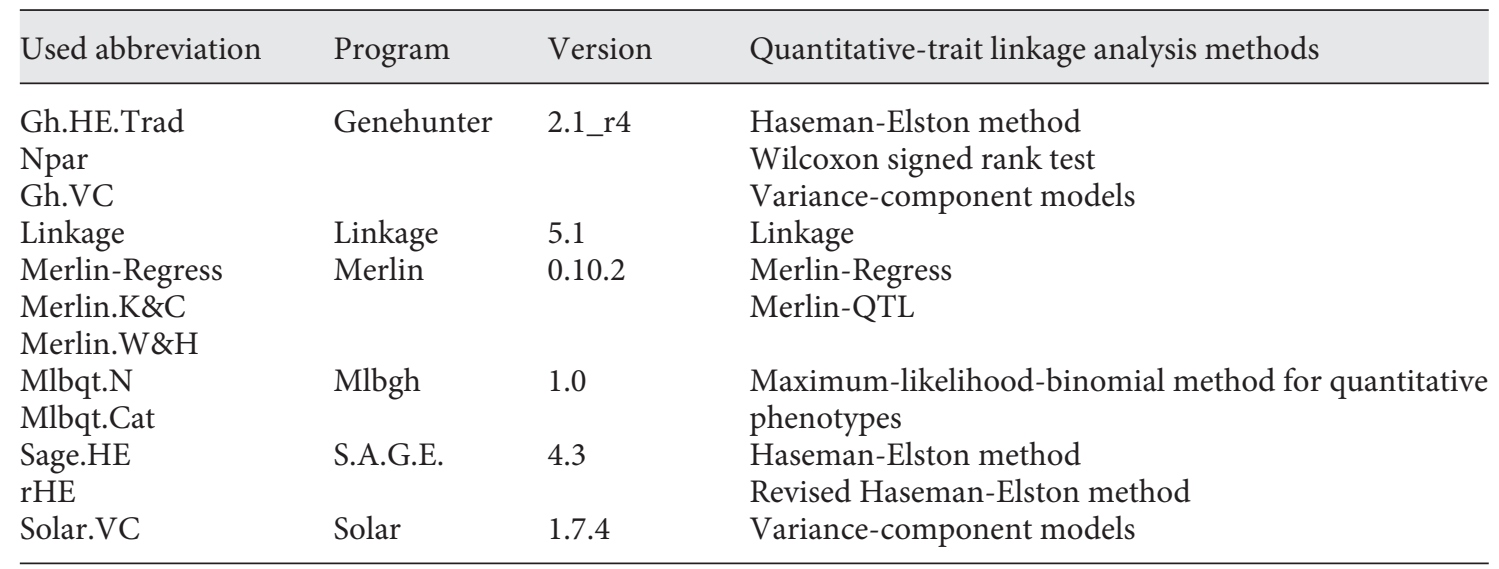

tended to dependent sibships, it is necessary to consider this point too [10]. Here, families are typically ascertained either at random, yielding the random sib-pair (RSP) design, or ascertained by one phenotypically extreme sibling - the study design is termed single proband sib-pair (SPSP) design, or ascertained by two phenotypically extreme siblings, the extreme sib-pair (ESP) design (for an overview, see, e.g., [11]). Both the SPSP and the ESP design can be expected to have greater power for mapping QT by linkage than the RSP design (see, e.g., [12]). Furthermore, sib-pairs where both siblings are phenotypically extreme are generally more powerful than sib-pairs ascertained by a single proband in single major gene models [13].

Among the statistical approaches for linkage mapping, variance component models (VC) received great attention in the past decade because they explicitly allow estimation of major gene effects, polygenic components as well as of environmental effects $[14,15]$. This explicit modeling relies on the assumption of multivariate normality, and if this assumption is not met, the test statistics do not keep their nominal levels. For example, Allison and colleagues [16] observed an empirical significance level of about $18 \%$ for specific genetic models if the nominal level was $5 \%$. This is especially important for selected samples because they exhibit a deviation from normality as shown by Dolan [17].

For other QT mapping approaches that seem to be more robust with respect to selected samples and/or deviation from normality, however, only very limited information is available. In all but one study, only two different statistical approaches have been compared by Monte-
Carlo simulations [18-22], and only two comparisons are available under specific ascertainment schemes [23, 24]. Analytical considerations are rare [18, 20, 25, 26]. Since the available comparisons are based on different sets of assumptions it is difficult to compare or combine them. For some methods including Merlin-QTL [27] and the nonparametric (NPAR) linkage method [28] the situation is even worse because analyses of robustness or power for these methods are completely lacking. Moreover, the effect of violation of multivariate normality assumptions and/or the effect of analyzing selected samples is only known for a limited number of approaches (for a discussion, see next section). Therefore, many authors urgently called for extensive additional studies for gaining a better understanding of QT mapping approaches regarding robustness and power under a wide range of conditions for a variety of study designs and models violating the assumption of normality [10, p 220-221; 16, p 541; 19, p 252; 23, p 872; 24, p 884].

To fill this gap, we compared eight commonly applied QT mapping methods as implemented in six packages (table 1) that are freely available for non-commercial purposes in a Monte-Carlo simulation study. We studied the robustness and power under a wide range of models and study designs. Specifically, we considered three genetic models (dominant, additive, recessive), three selection schemes (RSP, SPSP, ESP), two family structures (nuclear families with one sib-pair, varying number of sib-pairs per sibship) and the possible effect of deviation from normality (normal error, log-normal error). This yielded a total of 36 different simulation scenarios under the null hypothesis of no linkage and another 36 scenarios under 
the alternative hypothesis of linkage. To investigate the behavior of the test statistics under appropriately small significance levels, we generated 100,000 replicates under $\mathrm{H}_{0}$ and 1,000 replicates under $\mathrm{H}_{1}$ per model. The high number of replicates under $\mathrm{H}_{0}$ also allowed the calculation of significance thresholds from empirical distributions for investigating empirical power as recommended by Yu et al. [22].

This article is organized as follows: in the next section we give a brief introduction to common QTL mapping methods followed by the material and methods section with a detailed description of this simulation study and compared QTL mapping. The results are explained as a whole and for each method separately, followed by a thorough discussion.

\section{Common Quantitative Trait Linkage Mapping Methods}

In this section we sketch common methods for mapping QT by linkage. For a detailed overview the interested reader is referred to the literature [11, 29]. The HasemanElston method [HE] is the root of modern model-free linkage approaches for QT [30]. The basic idea relies on the principle of similarity which traces back to Penrose [31]: genetic similarity should imply phenotypic similarity. The most commonly used measure of genetic similarity among a sib-pair is the number of alleles shared identical by descent (IBD), and Haseman and Elston used the squared trait difference to measure phenotypic similarity. The standard statistical test for linkage is a simple linear regression of the squared trait differences on the proportion of alleles shared IBD. Because of its simplicity and robustness against deviations from the assumption of normality, the HE is still commonly applied in practice.

In the last two decades the classical HE has been extended in several ways (for an overview, see, e.g., special issue Hum Hered 2003:55 and [11]). A major point of improvement is based on the fact that the squared trait difference does not utilize all phenotypic information available [32-34]. Wright suggested the combination of the trait difference and the trait sum which retain the total information [34]. He specifically proposed to use the difference between the mean-corrected squared trait sum and the squared trait difference; an expression which is equivalent to four times the mean corrected cross-product. The resulting approach is termed Haseman-Elston method revisited (rHE) [35]. $\mathrm{rHE}$ has greater power than $\mathrm{HE}$ if the residual sib correlation $\rho$ is weak, but lower power if $\rho$ is high $[36,37]$. An interesting aspect of rHE is that it shows correct type I error fractions even under a model violating normality assumptions, strong residual correlation and an ESP ascertainment strategy [19]. Both HE and rHE were originally developed for independent sib-pairs, and the methods therefore tend to be liberal if they are applied to large sibships without appropriate corrections [38].

Sham et al. [21] further improved HE and rHE and implemented their method (Merlin-Regress) in the Merlin software package [27]. They reversed the original HE method and regressed the IBD sharing on the squared trait sums and squared trait differences in a multivariate model. In contrast to the original HE, Merlin-Regress automatically allows inclusion of all relative pairs in a pedigree. It requires, however, population-based estimates of the mean, the variance and the heritability of the trait. The correct specification of these parameters is however challenging, especially in case of selected families or nonnormally distributed traits. If they are correctly specified, Merlin-Regress reveals correct type I error fractions for normally as well as for non-normally distributed traits, even for varying sibship sizes as shown by Sham et al. in an extensive simulation study [21]. Moreover, type I error fractions were robust to ESP sampling. Therefore, Merlin-Regress seems to be applicable to both unselected and selected samples. Its power is comparable to VC [21], and therefore both Merlin-Regress and VC are more powerful than the original HE for normally distributed traits (see, e.g., [39]). Misspecification of exactly one of the parameters that need to be specified in Merlin-Regress results in a loss of power but not in a violation of the type I error fractions if the trait is normally distributed and if the samples are randomly ascertained [21]. However, it remains unclear whether this robustness of Merlin-Regress can be generalized to the more complex situation of selected samples and/or non-normal traits.

A second approach implemented in the software package Merlin is an extension to the framework of allele sharing statistics introduced by Whittemore and Halpern [40] and Kong and Cox [41]. As pointed out by Ferreira [29], this framework is most appropriate for the analysis of binary traits, but it has been adapted to QTs [27]. Instead of using the number of alleles shared IBD for scoring - which is commonly used in the affected sib-pair approach, the squared scores of all the founder alleles present in a specific inheritance vector are summed over all possible inheritance vectors. The score for each founder allele in a specific inheritance vector is calculated as the sum of the mean deviate for all individuals who carry that founder allele in the pedigree. The scores can be transformed in $\mathrm{z}$-scores in the usual way, and this results in the Whittemore and Halpern type test statistic 
(Merlin.W\&H). In addition, as proposed by Kong and Cox, a likelihood ratio test can be constructed, and this approach is abbreviated as Merlin.K\&C.

An approach with a somewhat similar flavor is the maximum-likelihood-binomial method for quantitative traits (Mlbqt) [18]. Mlbqt considers the whole sibship and relies on the idea of binomial distributions of parental alleles among offspring. A latent, i.e., unobserved binary variable $Y$ is introduced to capture the linkage information between the QT and the genetic marker. For each observed phenotypic value, $Y$ represents values 1 or 0 for affected and unaffected subjects respectively, and the probability for the latent variable $Y$ to be equal to 1 increases as the observed QT increases. Linkage is then investigated by a single parameter likelihood ratio test for all possible sets of $Y$ values within sibships weighted by their probabilities. Mlbqt can be applied for normally as well as non-normally distributed traits. In the latter case, the distributional assumption is replaced by the empirical distribution as a step-function defined from the cumulative frequencies, e.g., by use of empirical deciles. This also allows the application of Mlbqt to selected samples. As shown in a simulation study [18], Mlbqt generally has higher power than the classical HE, and it reveals correct type I error fractions for normally distributed traits.

Similar to Mlbqt for deciles, the model-free nonparametric linkage method for quantitative phenotypes (Npar) should also be robust to deviations from normality [28]. The authors proposed their method especially for situations where the phenotypes are non-normally distributed. The method is based upon a Wilcoxon signed rank test and is therefore applicable to any phenotypic distributions [28].

Apart from the model-free methods described before, the linkage program package can be used to run a fully parameterized LOD-Score analysis for QT mapping [42]. However, because for a complex disease the detailed inheritance model - with its allele numbers, their frequencies, genotypic means and variance - can hardly be specified, model-free mapping methods are almost always used.

\section{Material and Methods}

\section{Simulations}

Phenotypic Models

The phenotype simulation is based on a variance analytic model which is described in detail, e.g., by Falconer and Mackay [43]. The phenotypic value $x_{i k}$ of an individual $k$ within family $i$ is additively decomposed into an overall mean, a major gene effect $g_{i k}$ being determined by the genotype of a diallelic quantitative trait locus together with its specified inheritance model, an environmental effect $G_{i}$ simulated as family effect which assigns each member of the pedigree the same random value and, finally, an error term $\varepsilon_{i k}$ :

$$
x_{i k}=\mu+g_{i k}+G_{i}+\varepsilon_{i k}
$$

Datasets were simulated under dominant, additive and recessive genetic models with respective frequencies of the 'high allele' of $0.05,0.2$ and 0.3 . All models were simulated with a mean of 0 , a variance of 1 and heritability in a narrow sense of 0.5 . The total variance of 1 is decomposed into the variance of the major gene effect of 0.2 , variance of the environmental effect of 0.3 , and variance of the error term of 0.5 . The major gene and the environmental effects are simulated from a normal distribution. To assess the effect of a violation of normality, the error term was chosen either from a normal distribution or a three parametric lognormal distribution. To obtain a standard three parametric lognormally distributed variable we took the exponent of a standard normal variate, subtracted the mean of the standard lognormal distribution and, finally divided this by the standard deviation of the standard lognormally distributed variable.

Family Structures

Two different types of nuclear families were chosen as family structures. Within every dataset, we simulated either 300 families with two offspring (independent sibships) or a more realistic situation of 100 families with two, three, four and five sib-pairs per family and their respective proportions of 40, 30, 18 and 12\% (dependent sibships) according to Speer et al. [44].

\section{Genetic Marker}

One genetic marker with ten alleles with equal frequencies was simulated. Under $\mathrm{H}_{0}$ the genetic marker was simulated with a $\theta=0.5$ between genetic marker and QTL; under $\mathrm{H}_{1}$ we assumed complete linkage, i.e. $\theta=0$.

Selection of Families

To take into account different ascertainment schemes, the following constellations were simulated: We chose families without selection (random selection), families with at least one sibling in the highest quartile (single proband selection according to a SPSP design) and families with at least two siblings which were either both in the highest quartile, or both in the lowest quartile, or of which one was in the highest and one in the lowest quartile (double proband selection according to an ESP design).

Datasets

For the purpose of this article, we developed the software package SIBSIM [45] which is tailored for rapid simulation of QTs in nuclear families as well as in extended pedigrees. Specifically, we simulated 100,000 datasets under $\mathrm{H}_{0}$ and 1,000 datasets under $\mathrm{H}_{1}$. Entirely new data sets were generated for every model, selection scheme, family structure and phenotype distribution. Genotypes and phenotypes were simulated for all family members, thus no missing data were simulated. Altogether, we simulated 36 different models under both $\mathrm{H}_{0}$ and $\mathrm{H}_{1}$ (3 genetic models $\times 2$ family structures $\times 3$ selection schemes $\times 2$ distributions). 


\section{Implementations of QT Linkage Methods in Software}

Packages

Eight different QT methods for analyzing linkage implemented in six software packages were chosen that are freely available for non-commercial use. The methods, their corresponding programs, version numbers and abbreviations are displayed in table 1 . The analysis options are described below in detail. LOD scores were converted to $p$ values as described elsewhere [11, $p$ 159]. To assess possible implementation differences between different programs, the Haseman-Elston method and the variance component models were calculated with two different programs.

\section{Genehunter}

Genehunter (version 2.1_r4) [46] was used to calculate the HE method (Gh.HE.Trad), the Npar and the VC (Gh.VC). For all analyses we used the options 'all pairs unweighted' and 'no dominance variance'. Because parental genotypes were complete, we ignored the EM results.

\section{Linkage}

The linkage package (version 5.1) [42] was used to perform model-based quantitative-trait linkage analyses (Linkage). The true values for genotype means of the major gene effect, their corresponding frequencies of the diallelic QTL as well as populationbased parameters for variances of the phenotypes (for all models var $=1$ ) were employed for the analyses. The genotype means for the three different genetic models are given in table 2 , where the allele $A_{1}$ corresponds to the 'high allele'.

\section{Merlin}

The program Merlin-Regress which is a part of the Merlin program package (version 0.10.2) [21] was used to calculate the method proposed by Sham et al. (Merlin-Regress). The true population-based values for phenotypic mean, variance and heritability were applied for the analyses (for all models mean $=0$, variance $=$ 1 and heritability $=0.5$ ). To assess a possible influence of oneparameter model misspecification, all datasets were additionally analyzed under a wide range of parameter values for mean (range: \pm 5 ), variance (range: $0.1-10$ ) and heritability (range: 0.05-0.95). Merlin was also used for calculating Merlin-QTL: We evaluated both the Whittemore and Halpern (Merlin.W\&H) and the Kong and Cox (Merlin.K\&C) test statistics.

\section{Mlbgh}

The program Mlbgh (version 1.0) [18, 47] is a modification of Genehunter with the implementation of Mlbqt [18]. The analyses were performed both under the assumption of a standard normal distribution for the phenotypes (Mlbqt.N) and by defining a step function to divide the phenotypes in empirical deciles (Mlbqt. Cat).

\section{S.A.G.E.}

The program package S.A.G.E. (version 4.3) was used to calculate the HE (Sage.HE) and the rHE. The analyses were performed using the standard parameters, and for rHE the population-based true mean value was set to the correct value of 0 .

\section{Solar}

Solar (version 1.7.4) [14] was used to calculate VC (Solar.VC). The analyses were performed under the given default parameters.
Table 2. Genotype means for the major gene effect as used for the model-based analyses with the linkage program

\begin{tabular}{llcl}
\hline Model & $\mathrm{E}\left(\mathrm{A}_{2}, \mathrm{~A}_{2}\right)$ & $\mathrm{E}\left(\mathrm{A}_{1}, \mathrm{~A}_{2}\right)$ & $\mathrm{E}\left(\mathrm{A}_{1}, \mathrm{~A}_{1}\right)$ \\
\hline Dominant & -0.147 & 1.361 & 1.361 \\
Additive & -0.316 & 0.474 & 1.265 \\
Recessive & -0.141 & -0.141 & 1.422 \\
\hline
\end{tabular}

The allele $\mathrm{A}_{1}$ corresponds to the 'high allele'.

\section{Empirical Type I Error and Empirical Power}

For every model and method we investigated the robustness by estimating the empirical type I error at nominal type I error levels of 5,1 and $0.1 \%$ under $\mathrm{H}_{0}$. For power comparisons, the power was calculated as empirical power at an empirical type I error of 0.05 .

\section{Results}

\section{Type I Error}

The empirical type I error fractions at a nominal type I error of $1 \%$ are displayed in table 3 , and corresponding tables for the 0.1 and 5\% nominal significance levels are given in the online suppl. tables 5, 6 (for all online suppl. material, see www.karger.com/doi/10.1159/000289596). Under normality assumptions, no selection and independent sibships, VC were too liberal while Merlin.W\&H and linkage were conservative for almost all scenarios considered. The other methods showed the correct type I error fraction. A violation of one or more of those assumptions affected the type I error level for many of the methods. This is described below in detail for each method.

We note that the relative increase of a violation of the type I error fraction increases with decreasing nominal type I error. Specifically, at a nominal type I error of 5\%, independent randomly ascertained sib-pairs, a dominant genetic model and a normally distributed error term, VC showed an empirical type I error of $6.14 \%$, i.e., a relative inflation of $\sim 23 \%$. This increased to a relative inflation of $\sim 70 \%$ at the nominal $0.1 \%$ test level (empirical type I error level $0.170 \%$ ).

\section{Empirical Power}

The empirical power estimates at an empirical type I error fraction of 5\% are shown in table 4 . VC and MerlinRegress showed high empirical power for all models considered if the normality assumption was met. How- 
Table 3. Empirical type I error fractions at a nominal type I error fraction of $1 \%$

\begin{tabular}{|c|c|c|c|c|c|c|c|c|c|c|c|c|c|c|c|c|c|c|c|}
\hline \multirow[t]{3}{*}{ Methods } & \multirow[t]{3}{*}{ Sibships } & \multicolumn{9}{|c|}{ Normality assumptions } & \multicolumn{9}{|c|}{ Violation of normality assumptions } \\
\hline & & \multicolumn{3}{|c|}{ random selection } & \multicolumn{3}{|c|}{ single selection } & \multicolumn{3}{|c|}{ double selection } & \multicolumn{3}{|c|}{ random selection } & \multicolumn{3}{|c|}{ single selection } & \multicolumn{3}{|c|}{ double selection } \\
\hline & & dom & add & rec & dom & add & rec & dom & add & rec & dom & add & rec & dom & add & rec & dom & add & rec \\
\hline Gh.HE.Trad & Independent & 1.0 & 1.0 & 1.0 & 1.0 & 1.0 & 1.0 & 1.0 & 1.0 & 1.1 & 0.6 & 0.6 & 0.6 & 0.7 & 0.7 & 0.7 & 0.6 & 0.6 & 0.6 \\
\hline Sage.HE & & 1.0 & 1.0 & 0.9 & 1.0 & 1.0 & 0.9 & 1.0 & 1.0 & 1.1 & 0.7 & 0.7 & 0.7 & 0.7 & 0.7 & 0.7 & 0.7 & 0.7 & 0.7 \\
\hline rHE & & 1.0 & 1.0 & 1.0 & 1.0 & 1.0 & 0.9 & 1.0 & 1.0 & 1.0 & 1.0 & 0.9 & 0.9 & 0.9 & 0.9 & 0.9 & 1.0 & 0.9 & 0.9 \\
\hline Merlin-Regress & & 1.0 & 1.0 & 1.0 & 1.0 & 1.0 & 1.0 & 1.0 & 1.0 & 1.0 & 0.8 & 0.8 & 0.8 & 0.7 & 0.8 & 0.8 & 0.8 & 0.8 & 0.9 \\
\hline Solar.VC & & 1.4 & 1.1 & 1.4 & 0.7 & 0.4 & 0.2 & 2.1 & 2.0 & 2.2 & 6.3 & 5.8 & 5.2 & 0.4 & 0.2 & 0.1 & 5.5 & 5.6 & 4.9 \\
\hline Npar & & 1.0 & 1.0 & 1.0 & 1.0 & 0.9 & 1.0 & 1.0 & 1.0 & 1.0 & 1.0 & 1.0 & 0. & 1.0 & 1.0 & 1.1 & 1.0 & 1.0 & 1.0 \\
\hline Merlin.K\&C & & 1.0 & 1.0 & 1.0 & 1.0 & 0.9 & 0.9 & 1.0 & 1.0 & 1.0 & 1.0 & 1.0 & 0.9 & 1.0 & 1.0 & 1.0 & 1.0 & 1.0 & 1.0 \\
\hline Merlin.W\&H & & 0.7 & 0.7 & 0.7 & 0.6 & 0.6 & 0.6 & 0.6 & 0.7 & 0.7 & 0.7 & 0.7 & 0.6 & 0.7 & 0.6 & 0.7 & 0.7 & 0.6 & 0.7 \\
\hline Mlbqt.Cat & & 1.1 & 1.1 & 1.0 & 1.0 & 1.1 & 1.0 & 1.0 & 1.1 & 1.0 & 1.0 & 1.0 & 1.0 & 1.0 & 1.0 & 1.0 & 1.0 & 1.0 & 1.0 \\
\hline Linkage & & 0.4 & 0.0 & 0.2 & 1.0 & 0.2 & 0.8 & 1.0 & 0.5 & 0.9 & 0.6 & 0.0 & 0.3 & 1.1 & 0.3 & 1.1 & 1.1 & 0.4 & 0.9 \\
\hline Gh.HE.Trad & Dependent & 1.1 & 1.1 & 1.1 & 1.1 & 1.1 & 1.1 & 1.1 & 1.1 & 1.0 & 0.9 & 0.9 & 1.0 & 1.0 & 0.9 & 1.0 & 0.9 & 0.9 & 0.9 \\
\hline Sage.HE & & 1.2 & 1.1 & 1.3 & 1.2 & 1.1 & 1.2 & 1.2 & 1.1 & 1.1 & 1.1 & 1.0 & 1.1 & 0.7 & 0.7 & 0.8 & 1.2 & 1.0 & 1.1 \\
\hline $\mathrm{rHE}$ & & 1.7 & 1.5 & 1.5 & 1.4 & 1.3 & 1.4 & 1.3 & 1.2 & 1.2 & 1.9 & 1.7 & 1.7 & 1.4 & 1.3 & 1.3 & 1.5 & 1.3 & 1.4 \\
\hline Merlin-Regress & & 1.3 & 1.3 & 1.2 & 1.2 & 1.2 & 1.3 & 1.2 & 1.2 & 1.2 & 1.3 & 1.2 & 1.2 & 1.2 & 1.2 & 1.2 & 1.2 & 1.2 & 1.2 \\
\hline Solar.VC & & 1.4 & 1.1 & 1.3 & 1.1 & 0.9 & 0.9 & 1.3 & 1.1 & 1.3 & 4.7 & 4.1 & 4.0 & 1.5 & 1.5 & 1.1 & 4.0 & 3.7 & 3.4 \\
\hline Npar & & 1.1 & 1.2 & 1.1 & 1.1 & 1.2 & 1.2 & 1.1 & 1.1 & 1.1 & 1.1 & 1.2 & 1.1 & 1.2 & 1.1 & 1.2 & 1.1 & 1.1 & 1.1 \\
\hline Merlin.K\&C & & 1.0 & 1.0 & 0.9 & 0.9 & 1.0 & 1.0 & 0.9 & 1.0 & 0.9 & 0.9 & 1.0 & 0.9 & 0.9 & 0.9 & 0.9 & 0.9 & 1.0 & 1.0 \\
\hline Merlin.W\&H & & 0.7 & 0.8 & 0.7 & 0.7 & 0.7 & 0.7 & 0.7 & 0.7 & 0.7 & 0.7 & 0.7 & 0.7 & 0.7 & 0.7 & 0.7 & 0.7 & 0.7 & 0.8 \\
\hline Mlbqt.Cat & & 1.0 & 1.0 & 1.0 & 1.0 & 1.0 & 1.1 & 0.9 & 1.0 & 0.9 & 1.0 & 1.0 & 1.0 & 1.0 & 1.0 & 1.0 & 1.0 & 1.0 & 1.0 \\
\hline Linkage & & 0.5 & 0.0 & 0.2 & 0.9 & 0.1 & 0.7 & 0.8 & 0.1 & 0.6 & 0.6 & 0.0 & 0.4 & 1.0 & 0.2 & 0.9 & 0.9 & 0.2 & 0.7 \\
\hline
\end{tabular}

Table 4. Empirical power at an empirical type I error fraction of 5\%

\begin{tabular}{|c|c|c|c|c|c|c|c|c|c|c|c|c|c|c|c|c|c|c|c|}
\hline \multirow[t]{3}{*}{ Methods } & \multirow[t]{3}{*}{ Sibships } & \multicolumn{9}{|c|}{ Normality assumptions } & \multicolumn{9}{|c|}{ Violation of normality assumptions } \\
\hline & & \multicolumn{3}{|c|}{ random selection } & \multicolumn{3}{|c|}{ single selection } & \multicolumn{3}{|c|}{ double selection } & \multicolumn{3}{|c|}{ random selection } & \multicolumn{3}{|c|}{ single selection } & \multicolumn{3}{|c|}{ double selection } \\
\hline & & dom & add & rec & dom & add & rec & dom & add & rec & dom & add & rec & dom & add & rec & dom & add & rec \\
\hline Gh.HE.Trad & Independent & 34 & 38 & 32 & 69 & 64 & 64 & 59 & 67 & 57 & 18 & 18 & 17 & 16 & 11 & 16 & 17 & 21 & 19 \\
\hline Sage.HE & & 34 & 38 & 32 & 69 & 64 & 64 & 59 & 67 & 57 & 18 & 18 & 17 & 16 & 11 & 16 & 17 & 21 & 19 \\
\hline $\mathrm{rHE}$ & & 24 & 28 & 26 & 60 & 56 & 60 & 63 & 60 & 62 & 28 & 32 & 29 & 60 & 47 & 55 & 42 & 29 & 47 \\
\hline Merlin-regress & & 42 & 49 & 40 & 79 & 76 & 79 & 76 & 77 & 80 & 41 & 38 & 35 & 54 & 41 & 54 & 50 & 51 & 58 \\
\hline Solar.VC & & 44 & 47 & 42 & 82 & 72 & 78 & 73 & 77 & 79 & 40 & 35 & 35 & 44 & 26 & 43 & 41 & 42 & 55 \\
\hline Npar & & 21 & 27 & 23 & 60 & 58 & 58 & 36 & 44 & 38 & 40 & 83 & 35 & 83 & 71 & 77 & 37 & 63 & 36 \\
\hline Merlin.K\&C & & 13 & 19 & 14 & 40 & 40 & 43 & 46 & 54 & 45 & 25 & 48 & 24 & 76 & 56 & 73 & 56 & 73 & 52 \\
\hline Merlin.W\&H & & 15 & 20 & 15 & 43 & 42 & 44 & 47 & 56 & 47 & 26 & 50 & 25 & 78 & 59 & 73 & 58 & 75 & 54 \\
\hline Mlbqt.Cat & & 21 & 23 & 19 & 44 & 45 & 41 & 49 & 54 & 49 & 30 & 36 & 24 & 64 & 52 & 51 & 55 & 69 & 55 \\
\hline Linkage & & 69 & 41 & 58 & 94 & 66 & 90 & 92 & 70 & 89 & 66 & 39 & 45 & 95 & 54 & 73 & 76 & 60 & 69 \\
\hline Gh.HE.Trad & Dependent & 40 & 43 & 36 & 65 & 60 & 57 & 51 & 56 & 48 & 22 & 27 & 23 & 27 & 22 & 25 & 30 & 23 & 28 \\
\hline Sage.HE & & 44 & 45 & 41 & 70 & 64 & 62 & 55 & 59 & 54 & 36 & 40 & 36 & 44 & 37 & 45 & 46 & 39 & 44 \\
\hline $\mathrm{rHE}$ & & 36 & 42 & 39 & 64 & 57 & 59 & 52 & 57 & 52 & 44 & 51 & 46 & 65 & 60 & 65 & 62 & 59 & 59 \\
\hline Merlin-regress & & 49 & 52 & 47 & 77 & 72 & 72 & 63 & 66 & 64 & 50 & 54 & 49 & 64 & 52 & 63 & 63 & 58 & 62 \\
\hline Solar.VC & & 51 & 53 & 48 & 77 & 71 & 73 & 63 & 67 & 65 & 43 & 50 & 46 & 58 & 45 & 57 & 58 & 49 & 59 \\
\hline Npar & & 24 & 31 & 26 & 56 & 49 & 50 & 39 & 43 & 37 & 43 & 86 & 41 & 81 & 87 & 75 & 61 & 88 & 58 \\
\hline Merlin.K\&C & & 16 & 23 & 18 & 43 & 40 & 36 & 30 & 41 & 28 & 25 & 52 & 25 & 66 & 72 & 59 & 48 & 64 & 42 \\
\hline Merlin.W\&H & & 19 & 25 & 20 & 47 & 42 & 39 & 32 & 44 & 30 & 27 & 56 & 27 & 69 & 75 & 63 & 52 & 67 & 47 \\
\hline Mlbqt.Cat & & 23 & 28 & 21 & 44 & 43 & 33 & 33 & 41 & 31 & 30 & 43 & 29 & 59 & 57 & 49 & 48 & 54 & 43 \\
\hline Linkage & & 69 & 49 & 59 & 89 & 64 & 80 & 83 & 62 & 75 & 66 & 51 & 51 & 89 & 62 & 72 & 81 & 57 & 67 \\
\hline
\end{tabular}


ever, for non-normal models, Npar, Merlin.W\&H and Merlin.K\&C generally performed better. Merlin-Regress and VC were superior over HE for all considered models. As expected, the highest power was observed for the dominant and the recessive model for the gold standard of comparison, the fully parameterized LOD score analysis. For Npar and the allele sharing approaches Merlin and Mlbqt the empirical power increased under deviation from normality but decreased for the other methods with some unsystematic exceptions. The results will be explained below in more detail for each method separately.

\section{HE and $r H E$}

Dependent sibships led to only slightly inflated type I error levels for HE in the Gh implementation; a remarkable inflation was observed for the GEE approach taken by S.A.G.E. for HE and rHE. The empirical type I error was almost not affected by selection for both methods. Violation of normality assumptions led to conservative type I levels for $\mathrm{HE}$ with the exception of Sage.HE under random or double selection. For rHe the type I error was almost not affected with the exception of dependent sibships where the type I error tended to inflate.

The empirical power was in general inferior compared to Merlin-Regress and VC for all considered models with some exceptions for rHE under violation of normality assumptions.

\section{Merlin-Regress}

The empirical type I error is not affected by selection but became clearly too liberal for dependent sibships and slightly too conservative under violation of normality assumptions.

Merlin-Regress showed in general a very good power comparable to VC. Under violation of normality assumptions the power was in general higher than with VC.

\section{VC}

Gh.VC and Solar.VC implementations showed no clear differences in empirical type I error and power. To increase the readability of the tables the results for the Gh.VC are not shown. VC did not show the correct type I error for any model, not even for normality assumptions and independent sibships. VC is unacceptably too liberal, especially under violation of normality assumptions. The effect of single or double selection heavily influenced the type I error in both directions. The effect was much stronger for independent sibships where in general the
VC was very conservative under single selection and unacceptably too liberal for double selection.

In general, $\mathrm{VC}$ showed a very good power, comparable to Merlin-Regress. Under violation of normality assumptions the power was generally lower than for Merlin-Regress.

\section{Npar}

For independent sibships Npar fitted the nominal type I error for all models very well. The empirical type I error was not affected by selection or violation of normality assumptions but tended to become slightly too liberal for dependent sibships.

Under normality assumptions the power was in general lower than for Merlin-Regress, VC, HE and rHe. Under violation of normality assumptions the situation changed: While without selection the power was very high and comparable to the power of VC and MerlinRegress Npar outperformed the other methods under single selection and double selection with the exception of independent sibships.

\section{Merlin.W\&H and Merlin.K\&C}

In general, Merlin.W\&H was too conservative for all models, while Merlin.K\&C fit the nominal type I error very well. The empirical type I error was not affected by ascertainment, violation of normality assumptions or dependent sibships.

Both methods generally showed a low power under normality assumptions. The situation became better under violation of normality assumptions, especially under single or double selection where Merlin.W\&H and Merlin.K\&C showed a better power than VC and MerlinRegress, comparable to Mlbqt.

\section{Mlbqt.N and Mlbqt.Cat}

Mlbqt.N and Mlbqt.Cat showed nearly identical results. To increase the readability of the tables the results for the Mlbqt.N are not shown. Mlbqt fitted the nominal type I errors for all models very well. The empirical type I error was therefore not influenced by either selection, violation of normality assumptions or dependent sibships.

Mlbqt showed a low power under normality assumptions even under selection. Under violation of normality assumptions, with random selection, the power was intermediate. However, the method performed much better under single or double selection resulting in an empirical power being higher than for $\mathrm{VC}$ and Merlin-Regress, even comparable to Merlin.K\&C. 


\section{Linkage}

With some rare exceptions Linkage showed extremely too conservative type I error levels for all models, even under normality assumptions, independent sibships and random selection. Under single or double selection the type I error increased in general. The impact of dependent sibships is unclear.

As expected, the fully parameterized LOD score analysis showed the highest power for the dominant and the recessive model. For the additive model the power was comparable or a little bit lower than for Merlin-Regress and VC.

\section{Effect of Model Misspecification for Merlin-Regress}

The effects of a one-parameter model misspecification are shown in online supplementary figures 1-3. A misspecification of one parameter only resulted in a loss of power but still showed correct type I error fractions: a misspecification of the phenotypic mean showed the highest effect on empirical power while an underestimation of the phenotypic variance had only a tiny effect. The effect of a heritability misspecification was small for realistic misspecifications but increased at the lower and upper border of the possible scale of values.

\section{Discussion}

In this Monte-Carlo simulation study we investigated the type I error and power of different quantitative trait linkage approaches which are, to the best of our knowledge, currently used to map QTs in nuclear families. We specifically investigated dependent sibships, three common ascertainment schemes for three major gene models and the impact of non-normally distributed residuals. There are two important differences to previous simulation studies: (a) while others studied the effect of deviation from normality by transforming the phenotype $[16,19,21$, $23,24]$, we used a log-normally distributed error term. This allowed preserving the major effect and the familial component, while at the same time the residual was strongly skewed and kurtotic. (b) Most simulation studies used 10,000 replicates for a specific model under $\mathrm{H}_{0}$. However, confidence intervals of $\mathrm{p}$ values are $\sim 1 \%$ wide at the nominal 5\% test level for this low number of replicates. Thus, lower nominal type I error levels cannot be investigated although $\mathrm{p}$ values $\sim 0.001$ are of great interest in linkage studies. We therefore generated 100,000 replicates for each scenario under $\mathrm{H}_{0}$, resulting in a $\sim 0.002$ length of the $95 \%$ confidence interval of the $\mathrm{p}$ value. It must be pointed out that these results can be generalized with certainty only to the conditions considered in this study.

Some results are well known and could be confirmed by this study; some are new and will be discussed in this section.

Our results indicate that the VC approach results in substantially inflated type I errors even for normally distributed residuals, realistic sample sizes and randomly drawn nuclear families. There are two reasons that may explain this unexpected finding. First, Ferreira [29] noted that the expected covariance matrix includes six free parameters. However, when applied to sib-pair data, these are not identifiable, and additional restrictions to retain identifiability are required. Moreover, the standard errors of variances are fourth order moments. And it is well known that statistics relying on fourth-order moments are unstable for relatively small sample sizes that characterize most real data (see, e.g., [48]).

Some recommendations are given in the literature to improve the robustness of the VC. For example a simple transformation of the phenotype by a function to obtain a like-normally distributed phenotype that is hopefully multivariate normally distributed. However, there is no guarantee that a transformation exists that will result in a multivariate normal distribution. For example, the nonnormally distributed phenotypes in our study could not be transformed to multivariate normality by any function. A second recommendation is to utilize robust estimators [49], but the utilization of robust estimators could result in a power decrease. A third option for selected samples is to incorporate the selection probabilities into the calculation of the likelihood, but this may reduce the power too.

In contrast to $\mathrm{VC}$, Merlin-Regress is very robust and the type I error fractions are not influenced by selected samples without the need of robust estimators or other pull-ups with the only disadvantage of a liberal type I error in the situation of dependent sibships, as demonstrated in this publication. If, as concluded by several authors $[21,22]$ and our results, VC and Merlin-Regress show nearly the same high empirical power, Merlin-Regress should be preferred to VC for mapping QTs in nuclear families with realistic sibship sizes as used in this study. Moreover, we demonstrated that the type I error of Merlin-Regress is not influenced by a one-parameter model misspecification and that only a minimal loss of power resulted even in the complex situations of selected data and non-normally distributed traits. However, as pointed out by one reviewer, model misspecification in a real scenario will result from a combination of various parameters and is much more complicated than the one-param- 
eter model used in this study. In addition Huang and colleagues identified at the GAW 15 a liberal type I error of Merlin-Regress if applied to extended pedigrees [50]. Further studies are needed to address these points in detail before a clear recommendation for Merlin-Regress can be given.

The next well studied approaches are HE and rHE. The remarkably lower power compared to Merlin-Regress and the strong influence of non-normally distributed residuals on the empirical type I error should make it unnecessary to use these methods anymore.

The allele sharing approach Merlin.W\&H was in generally too conservative for all models. An explanation is given by Kong \& Cox [41] where the authors showed that for approaches like the W\&H, a less than perfectly informative genetic marker can result in an unacceptable conservative type I error. The W\&H approach should therefore not be used. The other two allele sharing approaches Merlin.K\&C and Mlbqt were very robust but the empirical power is very low compared to other methods with some exceptions under non-normality and single or double ascertainment. Since this is the first comparison under violation of normality assumptions these results could not be generalized. The low power in comparison to the other methods prevents a clear recommendation for them. Mlbqt.Cat could still be a method of choice for some study designs if the phenotype is for example ordinal distributed and an empirical distribution could be defined.

The results for the Npar method were somewhat surprising. The method is robust like the allele sharing approaches with a small increase in type I-error for dependent sibships. Under normality assumptions the power was generally lower than for Merlin-Regress, VC, HE and $\mathrm{rHe}$ as expected for a nonparametric method. But surprisingly the situation changed under non-normal distribution. Npar showed a high power and outperformed the other methods under single selection and double selection with the exception of independent sibships. The remarkable power difference between the additive versus the dominant or recessive models under violation of normality can be explained by the chosen function for centering the number of alleles IBD in the test statistic as implemented in Gh. Kruglyak \& Lander recommend the implemented function especially to test for a QT with an additive effect $[28,51]$. For Npar and the allele sharing approaches the empirical power increased under deviation from normality but decreased for the other methods with some unsystematic exceptions. The reason could be the high third moment introduced by the log-normal distributed error term. The resulting distribution for each of dif- ferent genotypes of the diallelic QTL is highly right skewed but with the same expectation values like for the normal distributed error term. The allele sharing approaches and the nonparametric method seem to utilize this information in a better way than the other approaches.

Finally, the extremely conservative type I error levels for Linkage are well known. For example, Rao and colleagues showed for a large number of empirical studies that the observed type I error was considerably less than the nominal type I error [52]. The high power of linkage, as shown in this study, can almost not be archived in real data studies because the correct mode of inheritance can hardly be specified for a complex disease. Therefore, model-free mapping methods should almost always the method of choice.

\section{Electronic-Database Information}

URLs for data presented herein are as follows:

Genehunter, http://www.broadinstitute.org/ftp/distribution/ software/genehunter/

Linkage, ftp://linkage.rockefeller.edu/software/linkage

Merlin, http://www.sph.umich.edu/csg/abecasis/Merlin/ download/

Mlbgh, http://genamics.com/software/downloads/mlbgh-1.0. tar.Z

S.A.G.E., http://darwin.cwru.edu/sage/

SIBSIM, http://www.imbs-luebeck.de/download/software/index.php

Solar, http://solar.sfbrgenetics.org/download.html

References

1 Duggirala R, Williams JT, Williams-Blangero S, Blangero J: A variance component approach to dichotomous trait linkage analysis using a threshold model. Genet Epidemiol 1997; 14:987-992.

2 Blangero J: Localization and identification of human quantitative trait loci: King harvest has surely come. Curr Opin Genet Dev 2004; 14:233-240

-3 Rankinen T, Zuberi A, Chagnon YC, Weisnagel SJ, Argyropoulos G, Walts B, Perusse L, Bouchard C: The human obesity gene map: The 2005 update. Obesity (Silver Spring) 2006;14:529-644.

4 Perola M, Sammalisto S, Hiekkalinna T, Martin NG, Visscher PM, Montgomery GW, Benyamin B, Harris JR, Boomsma D, Willemsen G, Hottenga JJ, Christensen K, Kyvik KO, Sorensen TI, Pedersen NL, Magnusson PK, Spector TD, Widen E, Silventoinen K, Kaprio J, Palotie A, Peltonen L: Combined genome scans for body stature in 6,602 european twins: Evidence for common Caucasian loci. PLoS Genetics 2007;3:e97. 
5 Streeten EA, McBride DJ, Pollin TI, Ryan K, Shapiro J, Ott S, Mitchell BD, Shuldiner AR, O'Connell JR: Quantitative trait loci for BMD identified by autosome-wide linkage scan to chromosomes $7 \mathrm{q}$ and $21 \mathrm{q}$ in men from the Amish Family Osteoporosis Study. J Bone Miner Res 2006;21:1433-1442.

6 Zmuda JM, Sheu YT, Moffett SP: The search for human osteoporosis genes. J Musculoskelet Neuronal Interact 2006;6:3-15.

-7 Timmann C, Evans JA, König IR, Kleensang A, Rüschendorf F, Lenzen J, Sievertsen J, Becker C, Enuameh Y, Kwakye KO, Opoku E, Browne EN, Ziegler A, Nürnberg P, Horstmann RD: Genome-wide linkage analysis of malaria infection intensity and mild disease. PLoS Genetics 2007;3:e48.

$\checkmark 8$ Korstanje R, Paigen B: From QTL to gene: The harvest begins. Nat Genet 2002;31:235236.

-9 Terwilliger JD, Goring HH: Gene mapping in the 20th and 21st centuries: Statistical methods, data analysis, and experimental design. Hum Biol 2000;72:63-132.

$>10$ Feingold E: Regression-based quantitativetrait-locus mapping in the 21st century. Am J Hum Genet 2002;71:217-222.

11 Ziegler A, König IR: A statistical approach to genetic epidemiology : Concepts and applications. 2nd ed. Weinheim, Wiley-VCH, 2010.

-12 Blackwelder WC, Elston RC: A comparison of sib-pair linkage tests for disease susceptibility loci. Genet Epidemiol 1985;2:85-97.

13 Risch N, Zhang H: Extreme discordant sib pairs for mapping quantitative trait loci in humans. Science 1995;268:1584-1589.

14 Almasy L, Blangero J: Multipoint quantitative-trait linkage analysis in general pedigrees. Am J Hum Genet 1998;62:1198-1211.

15 Amos CI: Robust variance-components approach for assessing genetic linkage in pedigrees. Am J Hum Genet 1994;54:535-543.

-16 Allison DB, Neale MC, Zannolli R, Schork NJ, Amos CI, Blangero J: Testing the robustness of the likelihood-ratio test in a variancecomponent quantitative-trait loci-mapping procedure. Am J Hum Genet 1999;65:531544.

-17 Dolan CV, Boomsma DI: Optimal selection of sib pairs from random samples for linkage analysis of a QTL using the EDAC test. Behav Genet 1998;28:197-206.

- 18 Alcaïs A, Abel L: Maximum-likelihood-binomial method for genetic model-free linkage analysis of quantitative traits in sibships. Genet Epidemiol 1999; 17:102-117.

- 19 Allison DB, Fernández JR, Heo M, Beasley TM: Testing the robustness of the new Haseman-Elston quantitative-trait loci-mapping procedure. Am J Hum Genet 2000;67:249252.
20 Sham PC, Purcell S: Equivalence between Haseman-Elston and variance-components linkage analyses for sib pairs. Am J Hum Genet 2001;68:1527-1532.

21 Sham PC, Purcell S, Cherny SS, Abecasis GR: Powerful regression-based quantitative-trait linkage analysis of general pedigrees. Am J Hum Genet 2002;71:238-253.

22 Yu X, Knott SA, Visscher PM: Theoretical and empirical power of regression and maximum-likelihood methods to map quantitative trait loci in general pedigrees. Am J Hum Genet 2004;75:17-26.

23 Cuenco KT, Szatkiewicz JP, Feingold E: Recent advances in human quantitative-traitlocus mapping: Comparison of methods for selected sibling pairs. Am J Hum Genet 2003, 73:863-873

24 Szatkiewicz JP, Cuenco KT, Feingold E: Recent advances in human quantitative-traitlocus mapping: Comparison of methods for discordant sibling pairs. Am J Hum Genet 2003;73:874-885.

-25 Chen WM, Broman KW, Liang KY: Quantitative trait linkage analysis by generalized estimating equations: unification of variance components and Haseman-Elston regression. Genet Epidemiol 2004;26:265-272.

26 Ziegler A: The new Haseman-Elston method and the weighted pairwise correlation statistic are variations on the same theme. Biom J 2001;43:697-702.

27 Abecasis GR, Cherny SS, Cookson WO, Cardon LR: Merlin-rapid analysis of dense genetic maps using sparse gene flow trees. Nat Genet 2002;30:97-101.

28 Kruglyak L, Lander ES: A nonparametric approach for mapping quantitative trait loci Genetics 1995;139:1421-1428.

29 Ferreira MA: Linkage analysis: Principles and methods for the analysis of human quantitative traits. Twin Res 2004;7:513530.

30 Haseman JK, Elston RC: The investigation of linkage between a quantitative trait and a marker locus. Behav Genet 1972;2:3-19.

31 Penrose LS: Genetic linkage in graded human characters. Ann Eugen 1938;6:133-138.

\$2 Drigalenko E: How sib pairs reveal linkage. Am J Hum Genet 1998;63:1242-1245.

33 Fulker DW, Cherny SS: An improved multipoint sib-pair analysis of quantitative traits. Behav Genet 1996;26:527-532.

34 Wright FA: The phenotypic difference discards sib-pair QTL linkage information. Am J Hum Genet 1997;60:740-742.

35 Elston RC, Buxbaum S, Jacobs KB, Olson JM: Haseman and Elston revisited. Genet Epidemiol 2000;19:1-17.

36 Forrest WF: Weighting improves the 'new Haseman-Elston' method. Hum Hered 2001; 52:47-54.
37 Palmer LJ, Jacobs KB, Elston RC: Haseman and Elston revisited: the effects of ascertainment and residual familial correlations on power to detect linkage. Genet Epidemiol 2000;19:456-460.

38 Williams JT, Blangero J: Power of variance component linkage analysis to detect quantitative trait loci. Ann Hum Genet 1999;63: 545-563.

-39 Amos CI, Zhu DK, Boerwinkle E: Assessing genetic linkage and association with robust components of variance approaches. Ann Hum Genet 1996;60:143-160.

40 Whittemore AS, Halpern J: A class of tests for linkage using affected pedigree members. Biometrics 1994;50:118-127.

$\checkmark 41$ Kong A, Cox NJ: Allele-sharing models: LOD scores and accurate linkage tests. Am J Hum Genet 1997;61:1179-1188.

-42 Lathrop GM, Lalouel JM, Julier C, Ott J: Strategies for multilocus linkage analysis in humans. Proc Natl Acad Sci USA 1984;81: 3443-3446.

43 Falconer DS, Mackay TFC: Introduction to quantitative genetics, ed 4th. Essex, England, Longman, 1996.

44 Speer MC, Terwilliger JD, Ott J: Data simulation for GAW 9 problems 1 and 2. Genet Epidemiol 1995;12:561-564.

45 Franke D, Kleensang A, Ziegler A: IBSIM quantitative phenotype simulation in extended pedigrees. GMS Med Inform Biom Epidemiol 2006;2:Doc4.

46 Kruglyak L, Daly MJ, Reeve-Daly MP, Lander ES: Parametric and nonparametric linkage analysis: A unified multipoint approach. Am J Hum Genet 1996;58:1347-1363.

-47 Abel L, Müller-Myhsok B: Robustness and power of the maximum-likelihood-binomial and maximum-likelihood-score methods, in multipoint linkage analysis of affected-sibship data. Am J Hum Genet 1998;63:638647.

48 Bentler PM, Dudgeon P: Covariance structure analysis: Statistical practice, theory, and directions. Annu Rev Psychol 1996;47:563592.

49 Blangero J, Williams JT, Almasy L: Robust LOD scores for variance component-based linkage analysis. Genet Epidemiol 2000;19 (suppl 1):S8-S14.

50 Huang S, Ballard D, Zhao H: The role of heritability in mapping expression quantitative trait loci. BMC Proc 2007;1(suppl 1):S86.

51 Kruglyak L, Lander ES: Complete multipoint sib-pair analysis of qualitative and quantitative traits. Am J Hum Genet 1995;57: 439-454.

52 Rao DC, Keats BJ, Morton NE, Yee S, Lew R: Variability of human linkage data. Am J Hum Genet 1978;30:516-529. 